\title{
Monosporascus eutypoides, a Cause of Root Rot and Vine Decline in Tunisia, and Evidence that M. cannonballus and M. eutypoides Are Distinct Species
}

I. Ben Salem, Institut Supérieur Agronomique de Chott Mariem, Département des Sciences Biologiques et de la Protection des Plantes, 4042 Sousse, Tunisia; K. C. Correia, Universidade Federal Rural de Pernambuco, Departamento de Agronomia, 52171-900 Recife, Pernambuco, Brazil; N. Boughalleb, Institut Supérieur Agronomique de Chott Mariem, Département des Sciences Biologiques et de la Protection des Plantes, 4042 Sousse, Tunisia; S. J. Michereff, Universidade Federal Rural de Pernambuco, Departamento de Agronomia, 52171-900 Recife, Pernambuco, Brazil; and M. León, P. Abad-Campos, J. García-Jiménez, and J. Armengol, Instituto Agroforestal Mediterráneo, Universidad Politécnica de Valencia, Camino de Vera s/n, 46022-Valencia, Spain

\begin{abstract}
Ben Salem, I., Correia, K. C., Boughalleb, N., Michereff, S. J., León, M., Abad-Campos, P., García-Jiménez, J., and Armengol, J. 2013. Monosporascus eutypoides, a cause of root rot and vine decline in Tunisia, and evidence that $M$. cannonballus and M. eutypoides are distinct species. Plant Dis. 97:737-743.

Three Monosporascus eutypoides-like isolates recovered from cucurbit plants with symptoms of Monosporascus root rot and vine decline in Tunisia were compared to 28 isolates of M. cannonballus from 12 countries for phenotypic, genomic, and pathogenicity characteristics. Morphologically, M. cannonballus and M. eutypoides-like cultures were similar, each producing fertile perithecia in culture containing globose, smooth, dark brown to black ascospores. Nevertheless, all $M$. cannonballus isolates had one ascospore per ascus, while $M$. eutypoides-like isolates had mainly two to three ascospores per ascus (rarely one). The employment of the internal transcribed spacer (ITS) of nuclear ribosomal DNA, the elongation factor $1-\alpha(E F-1 \alpha)$, and the $\beta$-tubulin $(\beta-t u b)$ gene sequence diversity analyses and the resulting

phylogenies identified a level of polymorphism that enabled separation of $M$. cannonballus and $M$. eutypoides-like isolates. All isolates of $M$. cannonballus had identical $E F-1 \alpha$ and $\beta$-tub sequences irrespective of very diverse geographic origins, which were different from the $E F-1 \alpha$ and $\beta-t u b$ sequences of the M. eutypoides-like isolates (96 and $97 \%$ similarity, respectively). Similar results were obtained for the ITS region of rDNA. In addition, of three $M$. eutypoides-like isolates tested for pathogenicity, all three were pathogenic on watermelon, two were pathogenic on muskmelon, but only one was pathogenic on cucumber. The results demonstrate that the $M$. eutypoides-like isolates belong to the species $M$. eutypoides, and that M. cannonballus and M. eutypoides are distinct species.
\end{abstract}

Monosporascus root rot and vine decline of cucurbits is a common disease of muskmelon (Cucumis melo L.) and watermelon (Citrullus lanatus (Thunb.) Matsum. \& Nakai) crops in hot, semiarid to arid, as well as subtropical cucurbit-growing areas worldwide $(2,6,17)$. The soilborne ascomycetes Monosporascus cannonballus Pollack \& Uecker (21) and M. eutypoides (Petrak) von Arx (32) have been reported as causal agents of the disease (17). Nevertheless, it was suggested by some authors that both Monosporascus species might be conspecific $(13,17,23,24)$, as the species are similar in almost all morphological features except: in the number of mature ascospores in each ascus; both species have been reported on similar hosts causing root rot in almost identical climatic habitats; and there is no apparent sequence difference in their internal transcribed spacer (ITS) region of nuclear ribosomal DNA (nrDNA).

Monosporascus spp. received little attention from either mycologists or plant pathologists until recently. The genus Monosporascus Pollack \& Uecker was established by Pollack and Uecker in 1974 (21) with the type species $M$. cannonballus that caused decay of secondary roots on cantaloupe plants under greenhouse conditions in Arizona (30). M. cannonballus was characterized as having black, smooth perithecia in which clavate to pyriform asci containing one large (rarely two), spherical ascospores are produced $(21,23)$. Moreover, these authors indicated that they failed to get

Corresponding author: J. Armengol, E-mail: jarmengo@eaf.upv.es

I. Ben Salem and K. C. Correia contributed equally to this work.

Accepted for publication 8 December 2012.

http:dx.doi.org/10.1094/PDIS-05-12-0464-RE

(C) 2013 The American Phytopathological Society the ascospores to germinate, and that there was no known anamorph (21). The genus Monosporascus was similar to two species belonging to other genera of pyrenomycetes: Rechingeriella eutypoides Petrak, described from decayed roots of an unidentified plant in Pakistan (19), and Anixiella monospora Malloch \& Cain, which was described from a herbarium specimen of an Iris sp. rhizome from Iran (14). Both $R$. eutypoides and A. monospora produced large and spherical ascospores resembling those of $M$. cannonballus, but diverged morphologically in their perithecium structure, peridium type, ascus wall, and ascospore ornamentation (21,31). Subsequently, von Arx (32) transferred $R$. eutypoides into Monosporascus as M. eutypoides (Petrak) Arx. Additionally, he considered Bitrimonospora indica Sivanesan, Talde \& Tilak, a fungus found on roots of Achyranthes aspera, to be a synonym of $M$. eutypoides. $B$. indica has mostly two (rarely three) ascospores per ascus, which are capable of germinating by multiple germ tubes (25). Then, in 1978, Hawksworth and Ciccarone (10) transferred A. monospora into Monosporascus as another species, $M$. monosporus (Malloch \& Cain) D. Hawksw. \& A. Cicc.; however, some authors have been reluctant to accept this third species (17). At that time, there were only two recognized species in the genus Monosporascus, M. cannonballus and M. eutypoides, which were separated on the basis of the number and germinability of ascospores: M. cannonballus had one spore (rarely two) that did not germinate, and $M$. eutypoides had two spores (rarely three) that germinated readily (17).

In 1995, Lovic et al. (13) examined several cultures of M. cannonballus and $M$. eutypoides from the United States, Spain, and Japan for morphological and molecular characteristics. They only observed asci containing one ascospore, and the ascospores did not germinate in culture media. In addition, the ITS region of nrDNA from these isolates was amplified by polymerase chain reaction (PCR) assay, and no sequence difference was detected. Thus, the authors suggested two possible explanations: one, the two species 
are distinct but $M$. eutypoides no longer is a major component of the populations; and two, the two species are synonymous and some isolates might have been misidentified. The authors favored the synonymous hypothesis $(16,17)$. More recently, a new Monosporascus species, M. ibericus, isolated as an endophyte from roots and stems of three plant species growing on sand flats and salt marshes in the Ebro Delta (Spain), was described (7). The main characteristic of this species is the presence of a greater number of ascospores per ascus (up to six), compared to the other species of the genus.

During routine surveys of Monosporascus root rot and vine decline of cucurbits conducted in 2010 in Tunisia, several Monosporascus-like isolates were found which produced fertile perithecia in culture, containing asci with mainly two to three brown, spherical, smooth-walled ascospores which matched the description of $M$. eutypoides. Therefore, the objective of this research was to study these isolates by means of phenotypic characterization, DNA analysis, and pathogenicity tests in order to determine the taxonomic status.

\section{Materials and Methods}

Fungal isolates. This study included 28 isolates of $M$. cannonballus obtained from roots of different cucurbit hosts from different countries and continents, and one reference isolate of $M$. ibericus (CBS 110550) (Table 1). The isolates were obtained from: Z. Banihashemi (College of Agriculture, Shiraz University, Shiraz, Iran), B. D. Bruton (USDA, Lane, OK), A. Infantino (Istituto Sperimentale per la Patologia Vegetale, Rome, Italy), G. Quilosi (Facoltà di Agraria, Università degli Studi della Tuscia, Viterbo, Italy), A. Sarpeleh (Plant Protection Research Institute, Tehran, Iran), the Genetic Resources Center of the National Institute of Agrobiological Sciences (Tsukuba, Japan), the CBS Culture Collection (Utrecht, the Netherlands), the Culture Collection of Phytopathogenic Fungi "Prof. Maria Menezes" (CMM) of the Universidade Federal Rural de Pernambuco (Recife, Brazil), and the Culture Collection of the Instituto Agroforestal Mediterráneo (Valencia, Spain). In addition, three Monosporascus-like isolates obtained in Tunisia from roots of watermelon (MT45 and MT47) and cucumber (MT54) crops exhibiting symptoms of Monosporascus root rot and vine decline were evaluated. In previous observations, these isolates produced perithecia containing mainly two to three ascospores per ascus (unpublished data).

All isolates were hyphal-tipped and stored at $25^{\circ} \mathrm{C}$ in the dark in plastic vials containing sterilized peat (Gramoflor $\mathrm{GmbH} \& \mathrm{Co}$, Vechta, Germany) in a collection at the Laboratorio del Grupo de Investigación en Hongos Fitopatógenos (Instituto Agroforestal Mediterráneo, Universidad Politécnica de Valencia, Valencia, Spain). Prior to using the isolates, $0.1 \mathrm{~g}$ of the colonized peat from

Table 1. Isolates of Monosporascus spp. evaluated, with corresponding GenBank accession numbers and optimum growth temperatures $\left({ }^{\circ} \mathrm{C}\right)$

\begin{tabular}{|c|c|c|c|c|c|c|c|c|}
\hline \multirow[b]{2}{*}{ Species } & \multirow[b]{2}{*}{ Code } & \multirow[b]{2}{*}{ Source ${ }^{y}$} & \multirow[b]{2}{*}{ Location } & \multirow[b]{2}{*}{ Isolated from } & \multirow{2}{*}{$\begin{array}{l}\text { Opt. growth } \\
\text { temp. }\left({ }^{\circ} \mathrm{C}\right)^{\mathrm{z}}\end{array}$} & \multicolumn{3}{|c|}{ GenBank accession number } \\
\hline & & & & & & ITS & TUB & EF1 \\
\hline \multirow[t]{28}{*}{ M. cannonballus } & CMM2365 & $\mathrm{H}$ & Brazil, Icapui, Ceará & Muskmelon & $31.98 \pm 0.12$ & JQ762367 & JQ907308 & JQ958961 \\
\hline & CMM2386 & $\mathrm{H}$ & $\begin{array}{l}\text { Brazil, Pau Branco, Rio } \\
\text { Grande do Norte }\end{array}$ & Muskmelon & $31.44 \pm 0.14$ & JQ771917 & JQ907303 & JQ907318 \\
\hline & CMM2401 & $\mathrm{H}$ & Brazil, Quixeré, Ceará & Muskmelon & $31.81 \pm 0.13$ & JQ762362 & JQ907306 & JQ907313 \\
\hline & CMM2405 & $\mathrm{H}$ & Brazil, Quixeré, Ceará & Muskmelon & $32.20 \pm 0.12$ & JQ771916 & JQ907309 & - \\
\hline & CMM2429 & $\mathrm{H}$ & $\begin{array}{l}\text { Brazil, Mossoró, Rio } \\
\text { Grande do Norte }\end{array}$ & Muskmelon & $31.39 \pm 0.14$ & JQ762366 & JQ907311 & JQ907315 \\
\hline & CBS 586.93 & G & Egypt & Muskmelon & $33.15 \pm 0.13$ & JQ771930 & JQ907292 & JQ958948 \\
\hline & GT960051-1 & $\mathrm{B}$ & Guatemala & Muskmelon & $29.66 \pm 0.09$ & JQ771928 & JQ907293 & JQ958946 \\
\hline & GT980052-2 & $\mathrm{B}$ & Guatemala & Muskmelon & $30.55 \pm 0.05$ & JQ771932 & JQ907294 & JQ958947 \\
\hline & HON980076-2 & B & Honduras & Muskmelon & $30.82 \pm 0.05$ & JQ762394 & JQ973838 & JQ958931 \\
\hline & HON980085 & B & Honduras & Muskmelon & $30.51 \pm 0.07$ & JQ771929 & JQ973839 & JQ958932 \\
\hline & IR3 & $\mathrm{A}, \mathrm{E}$ & Iran, Yazd & Muskmelon & $32.66 \pm 0.16$ & JQ762363 & JQ907304 & JQ907312 \\
\hline & IR5 & $\mathrm{A}, \mathrm{E}$ & Iran, Kashan & Muskmelon & $32.82 \pm 0.14$ & JQ762361 & JQ907300 & JQ958895 \\
\hline & IS980126-1 & B & Israel & Muskmelon & $31.48 \pm 0.05$ & JQ762386 & JQ907295 & JQ958933 \\
\hline & 1325 & $\mathrm{C}$ & Italy, Sabaudia, Latina & Muskmelon & $31.29 \pm 0.08$ & JQ771920 & JQ907299 & JQ958953 \\
\hline & 1162 & $\mathrm{D}$ & $\begin{array}{l}\text { Italy, Pescia Romana, } \\
\text { Viterbo }\end{array}$ & Muskmelon & $30.86 \pm 0.20$ & JQ762371 & JQ907298 & JQ958951 \\
\hline & MC305550 & $\mathrm{F}$ & Japan & - & $32.36 \pm 0.11$ & JQ771918 & JQ973841 & JQ958954 \\
\hline & MC305581 & $\mathrm{F}$ & Japan & - & $30.78 \pm 0.09$ & JQ771922 & JQ903840 & JQ958955 \\
\hline & MC0199 & $\mathrm{I}$ & Portugal, Azambuja & Muskmelon & $31.94 \pm 0.14$ & JQ762387 & JQ973845 & JQ958936 \\
\hline & MC0399 & $\mathrm{I}$ & Portugal, Almeirim & Muskmelon & $31.84 \pm 0.25$ & JQ771911 & JQ973845 & JQ958938 \\
\hline & MC0498 & $\mathrm{I}$ & Spain & $\begin{array}{c}\text { Cucurbita } \\
\text { hybrid }\end{array}$ & $32.91 \pm 0.15$ & JQ762370 & JQ907305 & JQ907319 \\
\hline & MC0603 & I & Spain, Chilches, Castellón & Muskmelon & $32.14 \pm 0.05$ & JQ762364 & JQ907307 & JQ907314 \\
\hline & MC1103 & I & Spain, Meliana, Valencia & Muskmelon & $33.03 \pm 0.07$ & JQ762369 & JQ907302 & JQ907317 \\
\hline & MT38 & I & Tunisia, Sidi Bouzid & Watermelon & $31.89 \pm 0.12$ & JQ762365 & JQ907310 & JQ958960 \\
\hline & CA941029-3A & $\mathrm{B}$ & United States, California & Muskmelon & $30.72 \pm 0.02$ & JQ762391 & JQ907285 & JQ958915 \\
\hline & MC13 & $\mathrm{B}$ & United States, California & Muskmelon & $30.49 \pm 0.03$ & JQ743058 & JQ973842 & JQ958942 \\
\hline & MC30 & $\mathrm{B}$ & United States, Arizona & Muskmelon & $30.75 \pm 0.11$ & JQ762368 & JQ907301 & JQ907316 \\
\hline & TX902020-813 & $\mathrm{B}$ & United States, Texas & Muskmelon & $31.05 \pm 0.05$ & JQ743059 & JQ973837 & JQ958919 \\
\hline & TX923038-3C & B & United States, Texas & Muskmelon & $31.98 \pm 0.06$ & JQ958966 & JQ973843 & JQ958916 \\
\hline \multirow[t]{3}{*}{ M. eutypoides } & MT45 & $\mathrm{I}$ & Tunisia, Sidi Bouzid & Watermelon & $29.49 \pm 0.06$ & JQ958963 & JQ973834 & JQ958959 \\
\hline & MT47 & $\mathrm{I}$ & Tunisia, Sidi Bouzid & Watermelon & $29.38 \pm 0.07$ & JQ958964 & JQ973835 & JQ958956 \\
\hline & MT54 & I & Tunisia, Sfax & Cucumber & $29.38 \pm 0.07$ & JQ958964 & JQ973836 & JQ958956 \\
\hline M. ibericus & CBS 110550 & $\mathrm{G}$ & $\begin{array}{l}\text { Spain, Los Alfaques, } \\
\text { Tarragona }\end{array}$ & - & $27.62 \pm 0.20$ & JQ973832 & JQ973833 & JQ958958 \\
\hline
\end{tabular}

y Isolates were obtained from: A, Z. Banihashemi (College of Agriculture, Shiraz University, Shiraz, Iran); B, B. D. Bruton (USDA, Lane, OK, USA); C, A. Infantino (Istituto Sperimentale per la Patologia Vegetale, Rome, Italy); D, G. Quilosi (Facoltà di Agraria, Università degli Studi della Tuscia, Viterbo, Italy); E, A. Sarpeleh (Plant Protection Research Institute, Tehran, Iran); F, the Genetic Resources Center of the National Institute of Agrobiological Sciences (Tsukuba, Japan); G, the CBS Culture Collection (Utrecht, the Netherlands); H, the Culture Collection of Phytopathogenic Fungi "Prof. Maria Menezes" (CMM) of the Universidade Federal Rural de Pernambuco (Recife, Brazil); and I, the Culture Collection of the Instituto Agroforestal Mediterráneo (Valencia, Spain).

z Temperature growth data of each isolate were adjusted to a cubic polynomial model $\left(y=a+b x+c x^{2}+d x^{3}\right.$, where $y=$ optimal growth temperature, $x=$ mycelial growth, and $a, b, c$, and $d=$ regression parameters). Each value is the mean \pm standard error of 8 observations. 
each plastic vial was transferred to potato dextrose agar (PDA) (Biokar-Diagnostics, Zac de Ther, France) plates and maintained at $25^{\circ} \mathrm{C}$ in the dark for 10 days.

Phenotypic characterization. Perithecia formation in culture was determined for all isolates on PDA and V8-juice agar. Mycelial plugs ( $8 \mathrm{~mm}$ diameter) obtained from the growing edge of colonies were transferred to the center of PDA and V8-juice agar plates which were incubated for 2 months in the dark at 15, 20, 25, 30 , and $35^{\circ} \mathrm{C}$. There were four replicate plates for each isolate and temperature combination. All experiments were conducted twice.

For microscopic observations, single perithecia were mounted in $100 \%$ lactic acid (vol/vol) and observed using a Zeiss Axio Scope A.1 microscope. The diameter of 20 perithecia and 50 ascospores, and the length and width of 50 asci per isolate were measured using the imaging device Zeiss AxioVision LE. Photos were captured using a Zeiss AxioCam MRm digital camera from images recorded with the $\times 40$ objective.

Ascospore germination of isolates MT45, MT47, and MT54 was tested on tap-water agar (WA), PDA, and V8-juice agar at 25, 30, and $35^{\circ} \mathrm{C}(7,10)$. Perithecia were cut with a sterile blade to reveal the perithecial contents, and a drop of sterile water was placed on the cut surface of each fruiting body. A water drop containing masses of ascospores was then collected with a pipette from each perithecium, spread onto petri dishes containing the three culture media, and the spores dispersed with a Digralsky spreader (Deltalab, Barcelona, Spain). Plates were incubated in the dark at the different temperatures for 2 weeks. In addition, ascospore germination of these isolates was assessed in soil following the method described by Stanghellini et al. (26). Subsamples ( $7 \mathrm{~g}$ ) of non-autoclaved soil inoculated with ascospores $(1,000$ ascospores/g soil) were each dispensed into a propylene centrifuge tube $(8.0 \mathrm{~cm}$ long and $1.7 \mathrm{~cm}$ diameter). A total of five drainage holes (each $1 \mathrm{~mm}$ diameter) were drilled through the bottom of each tube prior to filling the tube with soil. Tubes were irrigated with sterile distilled water (SDW) until drainage occurred. A 2-day-old muskmelon seedling of the cultivar Temprano Rochet (Intersemillas, Quart de Poblet, Spain), pregerminated on moistened filter paper, was transplanted into each tube. Seeded tubes were placed in test-tube racks, transferred to growth chambers (12-h photoperiod), and incubated at 25 or $30^{\circ} \mathrm{C}$ for $1,5,10,15$, and 20 days. Subsequent to transplanting, all tubes were irrigated on alternative days with SDW until drainage occurred. At the termination of each experiment, each seedling was removed from the tubes, and the root system was washed gently in tap water and placed in a petri dish containing approximately $15 \mathrm{ml} \mathrm{SDW}$. The root system of each seedling was examined microscopically at $\times 40$ to $\times 400$ magnification to observe germinating ascospores attached to the secondary roots. All treatments were replicated three times and all germination experiments were conducted twice.

The effect of temperature on mycelial growth of each isolate was determined on PDA. Mycelial plugs $(8 \mathrm{~mm}$ diameter) obtained from the growing edge of colonies were transferred to the center of PDA plates which were kept in the dark at 5, 10, 15, 20, 25, 30, 35, or $40^{\circ} \mathrm{C}$. There were four replicates for each isolate and temperature combination. The diameter of each colony was measured perpendicularly in two directions when the colony reached at least two-thirds of the plate diameter, and the measurements were used to calculate the mean growth rate in $\mathrm{mm} /$ day. Additionally, after 15 days of incubation, colonies grown on PDA at $25^{\circ} \mathrm{C}$ were used to determine colony colors using the color charts of Rayner (22). Analyses of variance (ANOVA) were conducted with data obtained from temperature experiments to analyze potential trial-by-treatment interactions. ANOVA indicated that the data for the two repetitions were similar for each variable $(P>0.05)$, thus data from both repeats of the experiments were combined. For each isolate, average growth rates at each temperature were adjusted to a regression curve using Statgraphics Plus 5.1 (Manugistics Inc., Rockville, MD), and the best polynomial model was chosen based on parameter significance $(P<0.05)$ and coefficient of determination $\left(R^{2}\right)$ to estimate the optimum growth temperature of each isolate.

DNA isolation, sequencing, and phylogenetic analyses. Fungal mycelium was obtained from pure cultures grown in potato dextrose broth (PDB) (Sigma-Aldrich, Steinheim, Germany) for 3 weeks at $25^{\circ} \mathrm{C}$ in the dark. The mycelium was disrupted mechanically by grinding to a fine powder in liquid nitrogen using a mortar and pestle. Total DNA was extracted using the EZNA Plant Miniprep Kit (Omega Bio-tek, Norcross, GA) following the manufacturer's instructions. DNA was visualized on $1.5 \%$ agarose gels stained with ethidium bromide, and the DNA aliquots were stored at $-20^{\circ} \mathrm{C}$.

The ITS nrDNA region of each isolate was amplified using the universal primers ITS1F (8) and ITS4 (34). Fragments of the elongation factor $1-\alpha(E F-1 \alpha)$ and the $\beta$-tubulin $(\beta-t u b)$ genes were amplified using the primer pairs EF1-688F and EF1-1251R (1), and $\mathrm{T} 1$ (18) and $\mathrm{Bt} 2 \mathrm{~b}$ (9), respectively. Each PCR reaction contained $1 \times$ PCR buffer, $2.5 \mathrm{mM} \mathrm{MgCl} 2,200 \mu \mathrm{M}$ of each dNTP, 0.4 $\mu \mathrm{M}$ of each primer, $1 \mathrm{U}$ of DNA Taq polymerase (Dominion MBL, Córdoba, Spain), and $1 \mu \mathrm{l}$ of template DNA. The PCR reaction mix was adjusted to a final volume of $25 \mu \mathrm{l}$ with water (Chromasolv Plus, Sigma-Aldrich). PCR amplifications were performed on a Peltier Thermal Cycler-200 (MJ Research, Waltham, MA) using the following cycling: an initial denaturing step of $94^{\circ} \mathrm{C}$ for $2 \mathrm{~min}$; 35 cycles of $94^{\circ} \mathrm{C}$ for $30 \mathrm{~s}$, annealing at $55^{\circ} \mathrm{C}$ (for ITS region and $E F-1 \alpha$ fragment) or $50^{\circ} \mathrm{C}$ (for $\beta$-tub fragment) for $30 \mathrm{~s}$, and an extension at $72^{\circ} \mathrm{C}$ for $45 \mathrm{~s}$; and a final extension of $72^{\circ} \mathrm{C}$ for 10 min. The PCR products were visualized in $1.5 \%$ agarose gels (agarose D-1 Low EEO, Conda, Madrid, Spain) and molecular weights were estimated using the GeneRuler 100 bp Plus DNA Ladder (Fermentas, Carlsbad, CA).

PCR products were purified and sequenced in both directions by the DNA Sequencing Service of MCLAB (South San Francisco, CA) or Macrogen Inc., Sequencing Center (Seoul, South Korea). Sequences were edited using Sequencher Version 5.0 (Gene Codes Corporation, Ann Arbor, MI). The sequences were subjected to a BLAST search (http://www.ncbi.nlm.nih.gov/BLAST/) prior to phylogenetic analyses to identify the closest related sequences.

Table 2. Pathogenicity of Monosporascus spp. isolates to seedlings of muskmelon, watermelon, and cucumber 1 month after inoculation

\begin{tabular}{llccc}
\hline & & & Root disease severity $^{\mathbf{x}}$ \\
\cline { 3 - 5 } Species & Isolate & Muskmelon & Watermelon & Cucumber \\
\hline M. cannonballus & CMM2365 & $3.3^{\mathrm{y}} \mathrm{a}^{\mathrm{z}}$ & $1.5 \mathrm{ab}$ & $0.4 \mathrm{ab}$ \\
& MC1103 & $2.5 \mathrm{a}$ & $1.9 \mathrm{a}$ & $1.1 \mathrm{ab}$ \\
M. eutypoides & MT38 & $0.5 \mathrm{bc}$ & $0.4 \mathrm{bc}$ & $1.2 \mathrm{ab}$ \\
& MT45 & $1.5 \mathrm{abc}$ & $2.0 \mathrm{a}$ & $1.4 \mathrm{a}$ \\
MT47 & MT54 & $1.9 \mathrm{ab}$ & $1.9 \mathrm{a}$ & $1.3 \mathrm{ab}$ \\
& & $1.7 \mathrm{ab}$ & $1.7 \mathrm{ab}$ & $0.9 \mathrm{ab}$ \\
\hline
\end{tabular}

${ }^{\mathrm{x}}$ Root disease severity was evaluated on the following scale: $0=$ no discoloration or reduction of root mass; $1=$ slight discoloration with up to $30 \%$ root mass reduction; $2=$ moderate discoloration with 30 to $50 \%$ root mass reduction; $3=$ severe discoloration with 50 to $90 \%$ root mass reduction; and $4=$ root mass reduction $>90 \%$

${ }^{y}$ Values represent the means of 10 replications for each isolate, five per experiment with results pooled across the two repeats of the experiment.

${ }^{\mathrm{z}}$ Means in a column followed by the same letter do not differ significantly according to the Kruskal-Wallis nonparametric test $(P=0.05)$ 
Sequence alignment and phylogenetic analyses were conducted using Molecular Evolutionary Genetics Analysis (MEGA) Version 5 (28). Consensus sequences were aligned using CLUSTAL W (29). The best nucleotide substitution models for each data set were determined separately with the analysis option "Substitutions Model" in MEGA. The Kimura-2 parameter (11) was chosen as the best-fitting model for all data sets, and was used to search for best trees under the maximum likelihood criterion. The bootstrap test was performed with 1,000 repetitions to estimate the reliability of the tree. No outgroup was inserted in the analyses as the trees generated were unrooted.

Pathogenicity tests. Pathogenicity tests were conducted using three cucurbit species: cucumber cv. Edona (Royal Sluis, Enkhuizen, The Netherlands), muskmelon cv. Temprano Rochet, and watermelon cv. Fiera (Zeta Seeds, Benifayó, Spain); three isolates of M. cannonballus: CMM2365, MT38, and MC1103; and three isolates of $M$. eutypoides: MT45, MT47, and MT54 (Table 2). Inoculum was prepared by placing a fungal disk from a 2 -week-old PDA culture of the appropriate isolate into flasks containing autoclaved wheat kernels that had been immersed in distilled water for $12 \mathrm{~h}$. The flasks containing the wheat kernels were then incubated at $25^{\circ} \mathrm{C}$ for 4 weeks and shaken manually once a week. Six grams of colonized wheat kernels were mixed with enough sterilized peat moss to fill a $220 \mathrm{~cm}^{3}$ plastic pot, and a 2-week-old seedling (with one true leaf) of each cucurbit species was transplanted into each pot. The noninoculated control treatment consisted of plants grown in sterilized peat moss mixed with sterilized wheat kernels. All plants were maintained in a greenhouse for 1 month at 25 to $30^{\circ} \mathrm{C}$ in a completely randomized design, with five replications of each isolate and cucurbit species combination.

At the end of the experiment, the seedlings were carefully removed from the pots, and the root system of each seedling was washed gently in tap water and rated for disease severity based on the following scale adapted from Bruton et al. (4): $0=$ no discoloration or reduction of root mass; $1=$ slight discoloration with up to $30 \%$ root mass reduction; $2=$ moderate discoloration with 30 to $50 \%$ root mass reduction; $3=$ severe discoloration with 50 to $90 \%$ root mass reduction; and $4=$ root mass reduction $>90 \%$. Small pieces of representative root tissues of each seedling were surfacesterilized for $1 \mathrm{~min}$ in $1.5 \% \mathrm{NaOCl}$, washed twice with SDW, and plated onto PDA amended with $0.5 \mathrm{~g}$ streptomycin sulfate/liter. The experiment was conducted twice. The root disease severity caused by each isolate was analyzed with the Kruskal-Wallis nonparametric one-way ANOVA, and mean ranks of the treatment means were separated by a $Z$ test at $P<0.05$ using STATISTIX 9 (Analytical Software, Tallahassee, FL).

\section{Results}

Phenotypic characterization. All isolates of $M$. cannonballus produced perithecia when grown on PDA or V8-juice agar for 20 days at 25 and $30^{\circ} \mathrm{C}$. However, isolates MT38 and CMM2405 also produced perithecia on PDA at $20^{\circ} \mathrm{C}$; and isolates IR3, IR5, MT38, and CMM2405 produced perithecia on PDA at $35^{\circ} \mathrm{C}$, but only after 40 days of incubation (data not shown). M. eutypoides-like isolates MT45, MT47, and MT54 only produced perithecia on V8juice agar at 25 and $30^{\circ} \mathrm{C}$, while the isolate of $M$. ibericus failed to produce perithecia on either medium at any temperature tested (data not shown).

The morphological features of ascomata and sporulation of $M$. cannonballus isolates included the following: perithecia globose (250-) 487 (-710) $\mu \mathrm{m}$ diameter; asci clavate to pyriform, short stalked, thick-walled, and one spored, (37.5-) $44.3(-50.0) \times(57.5-)$ $71.5(-100.0) \mu \mathrm{m}$; and ascospores globose, smooth, dark brown to black, (32.5-) 40.9 (-47.5) $\mu \mathrm{m}$ diameter (Fig. 1A). For M. eu-

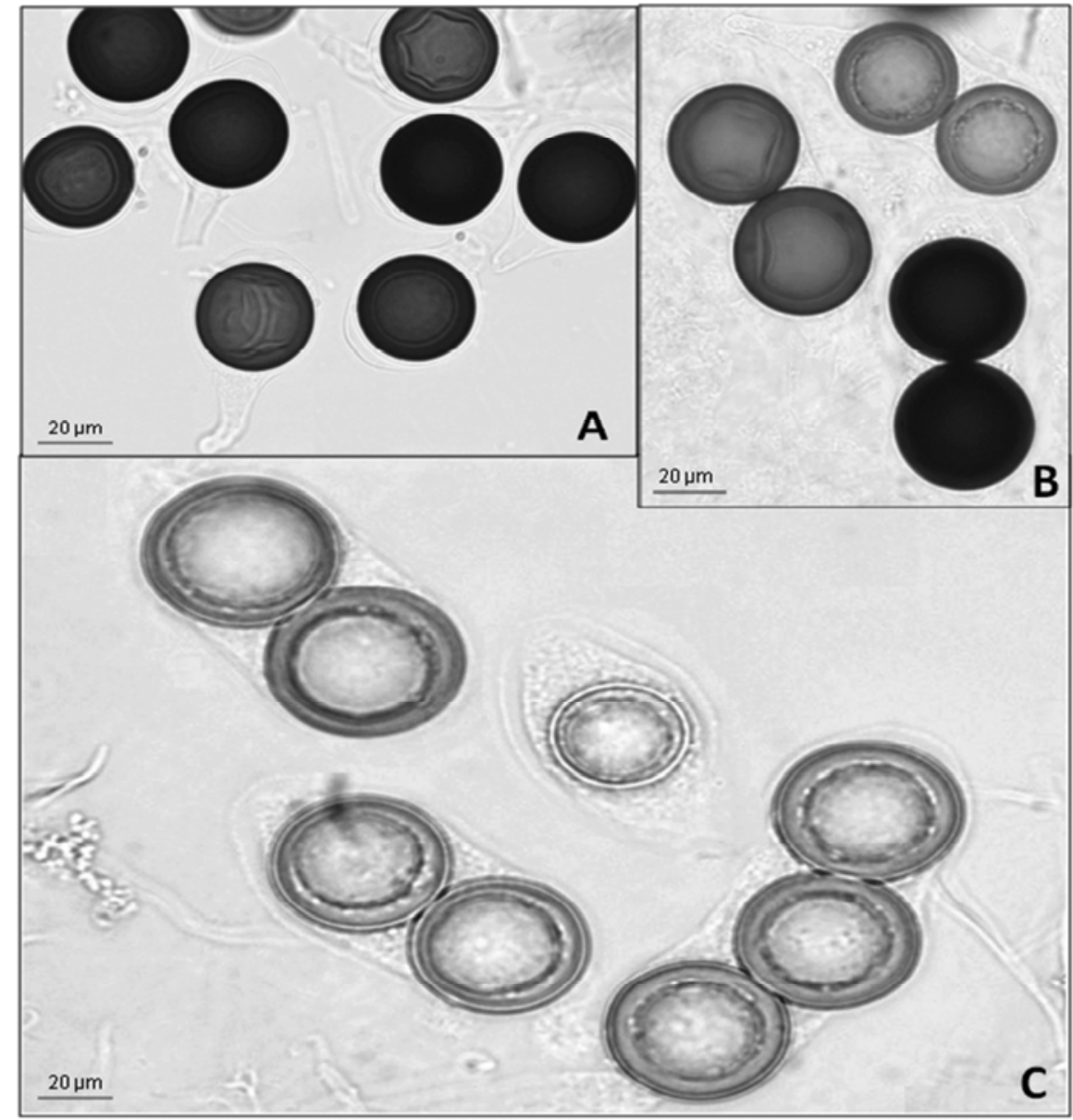

Fig. 1. A, Asci of Monosporascus cannonballus each containing a single ascospore. B and C, Asci of Monosporascus eutypoides each containing one to three ascospores. 
typoides-like isolates (MT45, MT47, and MT54), perithecia were globose and (400-) 598 (-940) $\mu \mathrm{m}$ in diameter; asci were cylindrical, short-stalked, varied in dimensions depending on whether there was one ascospore [(22.5-) $34.8(-65.5) \times(38.5-) 63.9$ $(-116.0) \mu \mathrm{m}]$, two ascospores [(21.5-) $35.1(-86.0) \times(49.0-) 75.6$ $(-199.0) \mu \mathrm{m}]$, or three ascospores [(21.0-) $34.7(-71.0) \times(56.0-)$ $90.1(-192.0) \mu \mathrm{m}]$, were mostly two-spored (rarely one- or threespored), and contained ascospores that were globose, smooth, dark brown to black, and (34.0-) $40.8(-47.0) \mu \mathrm{m}$ in diameter (Fig. 1B and $\mathrm{C}$ ).

Ascospore germination of $M$. eutypoides-like isolates was not observed on any of the three culture media used and at any of the incubation temperatures tested, but germination was observed (one to two germ tubes/ascospore) in experiments using the soil seedling assay after 15 and 20 days of incubation at $30^{\circ} \mathrm{C}$ (Fig. 2). All isolates of $M$. cannonballus grew on PDA at temperatures ranging from 10 to $40^{\circ} \mathrm{C}$; while isolates MT45, MT47, MT54, and $M$. ibericus only grew at temperatures of 5 to $35^{\circ} \mathrm{C}$. A cubic polynomial model $\left(y=a+b x+c x^{2}+d x^{3}\right.$, where $y=$ optimal growth temperature, $x=$ mycelial growth, and $a, b, c$, and $d=$ regression parameters) best fit temperature growth data for all isolates. The $R^{2}$ for the 28 regression equations ranged from 0.92 to 0.99 . Examples of these equations are: $y=22.6417-4.0253 x+0.2102 x^{2}-$ $0.0031 x^{3}$ for $M$. cannonballus isolate CMM2365, $y=4.1262-$ $1.2177 x+0.0994 x^{2}-0.0018 x^{3}$ for M. eutypoides isolate MT45, and $y=0.1210-0.1949 x+0.0326 x^{2}-0.0007 x^{3}$ for M. ibericus isolate CBS 110550 (the remaining 25 equations are not shown). Optimum growth temperatures for $M$. cannonballus (mean \pm standard error) ranged from $29.66 \pm 0.09^{\circ} \mathrm{C}$ (isolate GT960051-1) to $33.15 \pm 0.13^{\circ} \mathrm{C}$ (isolate CBS 586.93), with a mean value of $31.41 \pm$ $0.21^{\circ} \mathrm{C}$; while optimum growth temperatures for M. eutypoideslike isolates ranged from $29.38 \pm 0.07^{\circ} \mathrm{C}$ (isolates MT47 and MT54) to $29.49 \pm 0.06^{\circ} \mathrm{C}$ (isolate MT45), with a mean value of $29.43 \pm 0.03^{\circ} \mathrm{C}$. $M$. ibericus had an optimum growth temperature of $27.62 \pm 0.20^{\circ} \mathrm{C}$ (Table 1$)$. Colonies of $M$. cannonballus on PDA varied from buff (19"d) to avellaneous (17"'b) or olive (21"m) (22). Colonies of isolates MT45, MT47, and MT54 were smoke gray (21"'"d), and the isolate of M. ibericus was cinnamon (15") (22).

Sequencing and phylogenetic analyses. PCR products for the ITS, $\beta$-tub, and EF-1 $\alpha$ regions of the Monosporascus isolates were approximately 600,690 , and $650 \mathrm{bp}$, respectively. For M. cannonballus, the amplified sequences of $\beta$-tub and $E F-1 \alpha$ genes were identical for all isolates, while for the ITS nrDNA, all but 5 isolates (CMM2386, CMM2405, MC305550, GT980052-2, and GT960051-1) had identical ITS sequences. These five isolates each had a $\mathrm{G}$ insertion in a mononucleotide microsatellite in the ITS2 region (data not shown). The isolates MT-45, MT-47, and MT-54 had identical sequences at each of the three loci analyzed.

BLAST searches indicated that no $\beta-t u b$ and $E F-1 \alpha$ sequences similar to those of these Monosporascus spp. have been deposited in GenBank (data not shown). For the ITS region, the two sequences of $M$. cannonballus showed $100 \%$ identity with sequences of this species deposited in GenBank (accession numbers FJ430598 and AB097112). The maximum identity in the BLAST analysis with the sequence of isolates MT45, MT47, and MT54 was $95 \%$ similarity to the sequence of an unidentified soil fungus (EU479767). The ITS sequence of M. ibericus isolate CBS 110550 was $99 \%$ similar to the ITS sequence of an isolate of this species in GenBank (AF340013).

The ITS (including ITS1-5.8 S ITS2 rDNA sequence), $\beta$-tub, and $E F-1 \alpha$ sequence data sets consisted of 536, 652, and 610 characters after alignment. The size of the ITS aligned sequences was 512 or $513 \mathrm{bp}$ for all the M. cannonballus isolates; $526 \mathrm{bp}$ for isolates MT45, MT47, and MT54 of M. eutypoides; and 491 bp for the $M$. ibericus isolate. This variation in size was due to indels located mainly in the ITS1 region. The size of aligned sequences from the $\beta-t u b$ and $E F-1 \alpha$ genes showed limited variation: $\beta$-tub sequences were 644, 644, and $646 \mathrm{bp}$; and $E F-1 \alpha$ sequences were 600,600 , and 604 bp for all the M. cannonballus isolates, for iso- lates MT45, MT47, and MT54 of M. eutypoides, and for the $M$. ibericus isolate, respectively. The differences in the $\beta$-tub sequences were in three introns and two exons in the sequenced fragments. In the $E F-1 \alpha$ sequences, the variable sites were concentrated in the first intron (data not shown).

Results of ITS, $\beta$-tub, and $E F-1 \alpha$ phylogenetic analyses are shown in Figure 3. All phylogenies revealed three well-supported clades that contained the same isolates. Clade I grouped all $M$. cannonballus isolates, clade II contained the M. eutypoides-like isolates (MT45, MT47, and MT54), and clade III corresponded to the isolate of $M$. ibericus. Isolates in clades I and II were more closely related than the isolate in clade III.

Pathogenicity tests. Data for the repeated pathogenicity tests were combined because of the lack of significant differences between the two tests on each of muskmelon $(P=0.3452)$, watermelon $(P=0.4613)$, and cucumber $(P=0.1875)$. The statistical analyses indicated significant differences in root disease severity among isolates on all three cucurbit species evaluated $(P<0.0001)$ (Table 2). Of the six Monosporascus isolates tested for pathogenicity, two of three isolates of $M$. cannonballus and two of three isolates of M. eutypoides were pathogenic on muskmelon, with no significant differences in root disease severity among these four isolates. On watermelon, two of three $M$. cannonballus isolates, and all three $M$. eutypoides isolates were pathogenic compared to control plants, with no significant differences among the five pathogenic isolates. On cucumber, only one of three isolates of $M$. eutypoides and none of the M. cannonballus isolates was pathogenic. No isolate was pathogenic on all three cucurbit hosts. None of the M. cannonballus isolates was pathogenic on cucumber. Isolate MT38 was not pathogenic on any of the three hosts. All Monosporascus isolates were reisolated from roots of inoculated cucurbits, complying with Koch's postulates. No Monosporascus species were isolated from control plants.

\section{Discussion}

Monosporascus root rot and vine decline of cucurbits has been attributed to two Monosporascus species in the past, namely $M$. cannonballus and $M$. eutypoides $(17,21,32)$. In this study, three $M$. eutypoides-like isolates recovered from cucurbit plants with symptoms of Monosporascus root rot and vine decline in Tunisia were compared to 28 isolates of $M$. cannonballus from 12 countries on 4 continents. The phenotypic characterization showed similarities between $M$. cannonballus and $M$. eutypoides-like isolates. Isolates of both species produced fertile perithecia in culture with the perithecia containing globose, smooth, dark brown to black ascospores; and both had high optimum growth temperatures charac-

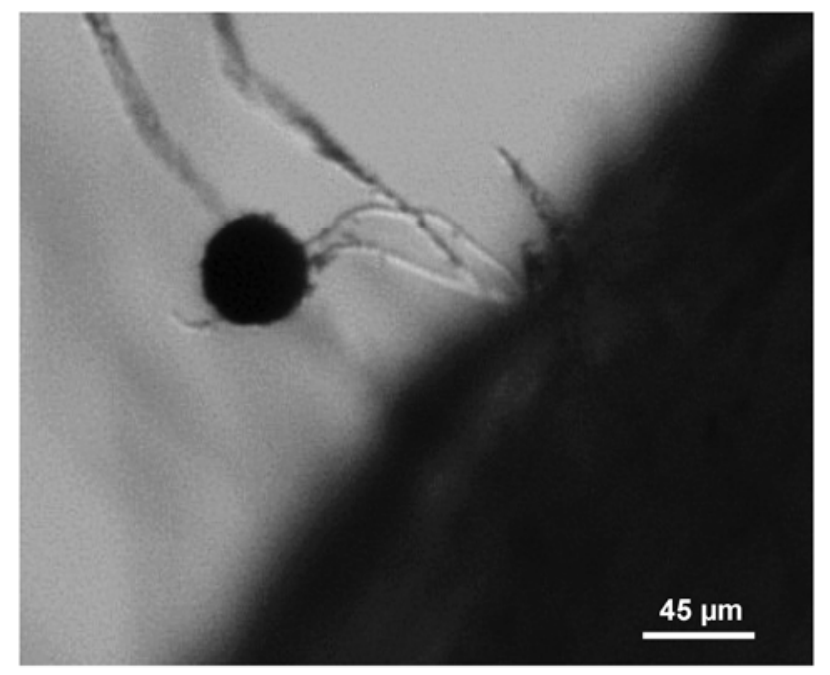

Fig. 2. Germinating ascospore of Monosporascus eutypoides incubated for 15 days at $30^{\circ} \mathrm{C}$, showing two germ tubes attached to a secondary root of a muskmelon seedling grown in soil inoculated with the fungus. 
teristic of Monosporascus spp. $(17,23,24)$. Nevertheless, all isolates of $M$. cannonballus had one ascospore/ascus, while $M$. eutypoides-like isolates had mainly two ascospores/ascus (rarely one or three).

Although Hawksworth and Ciccarone (10), and Martyn and Miller (17) indicated that perithecia and ascospore size may be variable for a single Monosporascus strain, depending on growth conditions and maturity of the spores, in general the measurements of perithecia, asci, and ascospores of both $M$. cannonballus and $M$. eutypoides-like isolates corresponded with those indicated for the species $M$. cannonballus and $M$. eutypoides, respectively $(17,23,24)$. It is interesting to note that, like other Monosporascus spp., none of the M. eutypoides-like isolates evaluated in this study exhibited an associated anamorph. Some additional differences were found between $M$. cannonballus and M. eutypoides-like isolates. M. cannonballus isolates had colonies buff to avellaneous or olive on PDA, which grew from 10 to $40^{\circ} \mathrm{C}$, and produced perithecia both in PDA and V8-juice agar; while $M$. eutypoides-like isolates were smoke gray on PDA, grew from 5 to $35^{\circ} \mathrm{C}$, and produced perithecia only on V8-juice agar.

In this study, germination of ascospores of M. eutypoides-like isolates could not be achieved in axenic culture, but the ascospores germinated readily in the rhizosphere of muskmelon plants growing in non-autoclaved field soil, as reported for M. cannonballus (26). Collado et al. (7) also were unable to observe germination of $M$. ibericus ascospores on corn meal agar, dichloran glycerol agar, malt-yeast agar, and PDA at 22, 28, and $37^{\circ} \mathrm{C}$. Hawksworth and Ciccarone (10) indicated that the ascospores of a species of Monosporascus isolated from Triticum in Lybia, which they identified as $M$. eutypoides based only on morphological features, germinated from 30 to $40^{\circ} \mathrm{C}$ by producing 5 to 10 germ tubes; however, these authors did not indicate on which culture media and under what incubation conditions the germination experiments were performed. As a result, all subsequent $M$. eutypoides descriptions have incorporated this morphological feature, which has only been observed for the isolate from Triticum, as characteristic of this species $(17,24)$.

Currently, the use of morphological characters alone is considered insufficient for species delimitation $(5,12)$. Thus, DNA sequence analyses were used for species identification in this study. The employment of ITS, $E F-1 \alpha$, and $\beta$-tub gene sequence diversity analyses with the resulting phylogenies made it possible to identify a level of polymorphism that enabled separation of isolates of $M$. cannonballus and M. eutypoides-like isolates, as well as an isolate of $M$. ibericus. The results demonstrate that all $M$. cannonballus isolates had identical $E F-1 \alpha$ and $\beta$-tub sequences, irrespective of diverse geographic origins, which were different from the $E F-1 \alpha$ and $\beta$-tub sequences of the M. eutypoides-like isolates. Similar results were obtained for the ITS region of nrDNA, except for a single nucleotide difference for 5 of the 28 isolates of M. cannonballus.

This is the first study in which a multigene analysis (ITS, $E F-1 \alpha$, and $\beta-t u b$ ) has been applied to a geographically wide collection of $M$. cannonballus isolates. To date, only ITS analysis had been used for the molecular characterization of Monosporascus spp. Lovic et al. (13) sequenced the ITS region of nine M. cannonballus isolates from Japan, Spain, and the United States, as well as three isolates from Spain, that were classified as $M$. eutypoides at the International Mycological Institute (Surrey, UK), with no apparent sequence differences detected among the 12 isolates. These authors pointed out that all of the isolates produced one ascospore/ascus, leading them to conclude that $M$. cannonballus and M. eutypoides were conspecific $(13,16,17)$. However, based on the morphological and molecular results of this study, it appears that the three $M$. eutypoides isolates examined by Lovic et al. (13) were misidentified, and all 12 isolates were, in fact, M. cannonballus. The same conclusion was published by Waugh et al. (33), who pointed out that "the isolate named as M. eutypoides employed in Lovic et al.'s study had one ascospore per ascus and did not germinate in axenic culture; it most closely fits the characteristics of $M$. cannonballus rather than $M$. eutypoides and was almost certainly misidentified". One of these "M. eutypoides" isolates from Spain (IMI 345135) remains at the CABI Culture Collection (UK). Unfortunately, efforts to revive this isolate were unsuccessful. Moreover, we have not been able to find additional cultures identified as M. eutypoides in other culture collections.

The results of this study strongly support the probability that the M. eutypoides-like isolates should be identified as M. eutypoides. The morphological characters fit with the description of this species, the isolates were obtained from cucurbit roots showing symptoms of Monosporascus root rot and vine decline, and each isolate was pathogenic to at least one of three cucurbit species tested. In
A: ITS

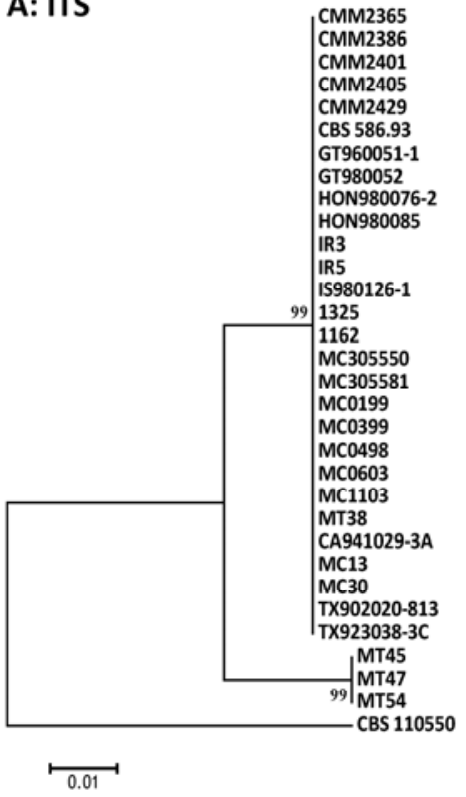

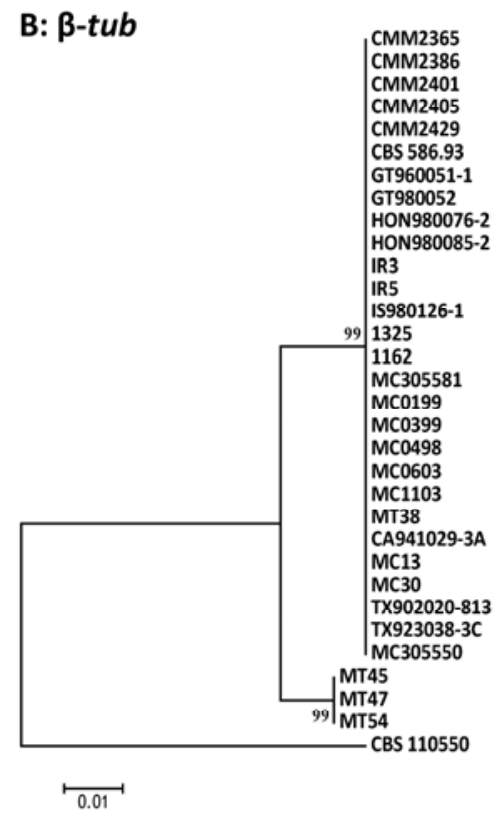

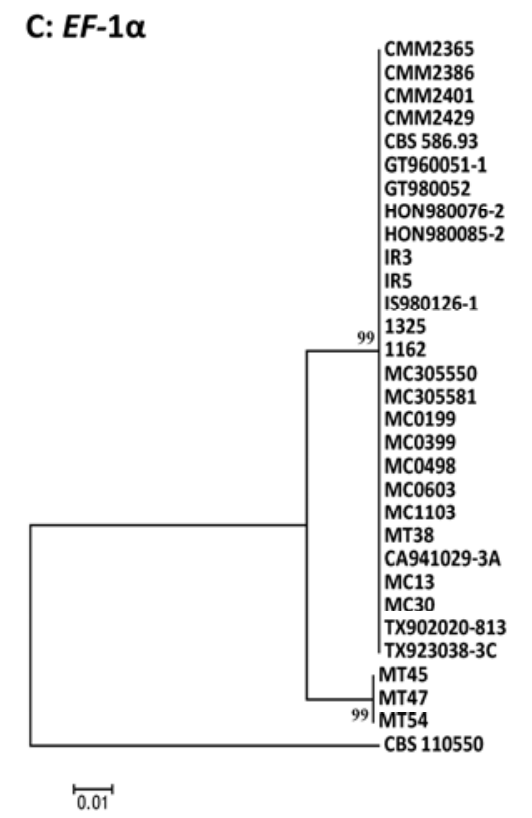

Clade I

Fig. 3. Unrooted phylogenetic trees from independent analyses of the A, internal transcribed spacer (ITS) region (536 bp), B, $\beta$-tubulin ( $\beta$-tub) (652 bp), and C, elongation factor 1-a (EF-1a) (610 bp) sequences of Monosporascus cannonballus, M. eutypoides, and $M$. ibericus isolates, inferred by using the Maximum Likelihood method based on Kimura-2 parameter model (11). Numbers in branches indicate the percent occurrence of the clade in 1,000 bootstrapped datasets. Analyses of ITS and $\beta$-tub sequences involved 32 nucleotide sequences, while EF-1a analysis was done with 31 sequences. Phylogenetic analyses were conducted in MEGA5 (28). 
addition, for the first time, based on multiple gene analyses, this study demonstrates that $M$. cannonballus and M. eutypoides are distinct species. Thus, the isolate MT47, obtained from watermelon roots in Tunisia, is designated as the epitype of the species $\mathrm{M}$. eutypoides. This isolate has been deposited at the CBS culture collection (CBS 132472).

Once $M$. eutypoides is confirmed as a distinct species associated with Monosporascus root rot and vine decline of cucurbits, further research is needed to determine the geographic distribution and host range of the species, and to improve identification methods. Both species coexist in Tunisian soils $(3,15)$ and perhaps also in other cucurbit growing countries. Thus, if the morphological features of M. cannonballus and M. eutypoides overlap, particularly for ascospore size, the extraction of ascospores from soil and quantification using a sucrose centrifugation technique (27) are not adequate to determine the presence of the two species in soil. Development of molecular techniques such as real time PCR methods (20) for early and accurate detection and quantification is, therefore, important.

\section{Acknowledgments}

We thank Z. Banihashemi (College of Agriculture, Shiraz University, Shiraz, Iran), B. D. Bruton (USDA, Lane, OK, USA), A. Infantino (Istituto Sperimentale per la Patologia Vegetale, Rome, Italy), G. Quilosi (Facoltà di Agraria, Università degli Studi della Tuscia, Viterbo, Italy), and A. Sarpeleh (Plant Protection Research Institute, Tehran, Iran) for providing strains of Monosporascus, without which this study would not have been possible. This research was supported financially by the Projects A/3878/05, A/5352/06, and A/017593/08 (Programa de Cooperación Científica e Investigación Inter-Universitaria entre España y Túnez, PCI-Mediterráneo, Ministerio de Asuntos Exteriores y Cooperación), and by CAPES (Project 203/2009 - International Cooperation CAPESBrazil/DGU-Spain). We are thankful to CAPES (Brazil) for the research fellowships granted to K. C. Correia and S. J. Michereff.

\section{Literature Cited}

1. Alves, A., Crous, P. W., Correia, A., and Phillips, A. J. L. 2008. Morphological and molecular data reveal cryptic speciation in Lasiodiplodia theobromae. Fungal Divers. 28:1-13.

2. Armengol, J., Alaniz, S., Vicent, A., Beltrán, R., Abad-Campos, P., GarcíaJiménez, J., Ben Salem, I., Souli, M., and Boughalleb, N. 2011. Effect of dsRNA on growth rate and reproductive potential of Monosporascus cannonballus. Fungal Biol. 115:236-244.

3. Boughalleb, N., Ben Salem, I., Beltrán, R., Vicent, A., Pérez-Sierra, A., Abad-Campos, P., García-Jiménez, J., and Armengol, J. 2010. Occurrence of Monosporascus cannonballus in watermelon fields in Tunisia and factors associated with ascospore density in soil. J. Phytopathol. 158:137-142.

4. Bruton, B. D., Garcia-Jimenez, J., Armengol, J., and Popham, T. W. 2000. Assessment of virulence of Acremonium cucurbitacearum and Monosporascus cannonballus on Cucumis melo. Plant Dis. 84:907-913.

5. Cabral, A., Rego, C., Nascimento, T., Oliveira, H., Groenewald, J. Z., and Crous, P. W. 2012. Multi-gene analysis and morphology reveal novel Ilyonectria species associated with black foot disease of grapevines. Fungal Biol. 116:62-80

6. Cohen, R., Pivonia, S., Crosby, K. M., and Martyn, R. D. 2012. Advances in the biology and management of Monosporascus vine decline and wilt of melons and other cucurbits. Hortic. Rev. 39:77-120.

7. Collado, J., González, A., Platas, G., Stchigel, A. M., Guarro, J., and Peláez, F. 2002. Monosporascus ibericus sp. nov., an endophytic ascomycete from plants on saline soils, with observations on the position of the genus based on sequence analysis of the 18S rDNA. Mycol. Res. 106:118-127.

8. Gardes, M., and Bruns, T. D. 1993. ITS primers with enhanced specificity for basiodiomycetes - Applications to the identification of mycorrhizae and rusts. Mol. Ecol. 2:113-118

9. Glass, N. L., and Donaldson, G. C. 1995. Development of primer sets designed for use with the PCR to amplify conserved genes from fila- mentous infection due to Phaeoacremonium spp. J. Clin. Microbiol. 41:1332-1336

10. Hawksworth, D. L., and Ciccarone, A. 1978. Studies on a species of Monosporascus isolated from Triticum. Mycopathology 66:147-151.

11. Kimura, M. 1980. A simple method for estimating evolutionary rate of base substitutions through comparative studies of nucleotide sequences. J. Mol. Evol. 16:111-120.

12. Lombard, L., Crous, P. W., Wingfield, B. D., and Wingfield, M. J. 2010. Multigene phylogeny and mating tests reveal three cryptic species related to Calonectria pauciramosa. Stud. Mycol. 66:15-30.

13. Lovic, B. R., Martyn, R. D., and Miller, M. E. 1995. Sequence analysis of the ITS regions of rDNA in Monosporascus spp. to evaluate its potential for PCR-mediated detection. Phytopathology 85:655-661.

14. Malloch, D., and Cain, R. F. 1971. New cleistothecial Sordariaceae and a new family, Coniochaetaceae. Can. J. Bot. 49:869-880.

15. Martyn, R. D., Lovic, B. R., Maddox, D. A., Germash, A., and Miller, M. E. 1994. First report of Monosporascus root rot/vine decline of watermelon in Tunisia. Plant Dis. 78:1220.

16. Martyn, R. D., Lovic, B. R., and Miller, M. E. 1993. Evidence that Monosporascus cannonballus and Monosporascus eutypoides may be synonymous. (Abstr.) Phytopathology 83:1347.

17. Martyn, R. D., and Miller, M. E. 1996. Monosporascus root rot and vine decline: An emerging disease of melons worldwide. Plant Dis. 80:716-725.

18. O'Donell, K., and Cigelnik, E. 1997. Two divergent intragenomic rDNA ITS2 types within a monophyletic lineage of the fungus Fusarium are not nonorthologous. Mol. Phylogenet. Evol. 7:103-116.

19. Petrak, F., and Ahmad, S. 1954. Beiträge zur Pilzflora Pakistans. Sydowia 8:162-185.

20. Picó, B., Roig, C., Fita, A., and Nuez, F. 2008. Quantitative detection of Monosporascus cannonballus in infected melon roots using real-time PCR. Eur J. Plant Pathol. 120:147-156.

21. Pollack, F. G., and Uecker, F. A. 1974. Monosporascus cannonballus: An unusual ascomycete in cantaloupe roots. Mycologia 66:346-349.

22. Rayner, R. W. 1970. A Mycological Colour Chart. Commonwealth Mycological Institute and British Mycological Society, Kew, Surrey, England.

23. Sivanesan, A. 1991. Monosporascus cannonballus. IMI Descriptions of Fungi and Bacteria No. 1035. Mycopathology 114:53-54

24. Sivanesan, A. 1991. Monosporascus eutypoides. IMI Descriptions of Fungi and Bacteria No. 1036. Mycopathology 114:55-56.

25. Sivanesan, A., Talde, U. K., and Tilak, S. T. 1974. Bitrimonospora indica gen. et sp. nov., a new loculoascomycete from India. Trans. Br. Mycol. Soc. 63:595-596.

26. Stanghellini, M. E., Kim, D. H., and Waugh, M. 2000. Microbe-mediated germination of ascospores of Monosporascus cannonballus. Phytopathology 90:243-247.

27. Stanghellini, M. E., and Rasmussen, S. L. 1992. A quantitative method for the recovery of ascospores of Monosporascus cannonballus from field soil. (Abstr.) Phytopathology 82:1115.

28. Tamura, K., Peterson, D., Peterson, N., Stecher, G., Nei, M., and Kumar, S 2011. MEGA5: Molecular Evolutionary Genetics Analysis using Maximum Likelihood, Evolutionary Distance, and Maximum Parsimony Methods. Mol. Biol. Evol. 28:2731-2739.

29. Thompson, J. D., Higgins, D. G., and Gibson, T. J. 1994. CLUSTAL W: Improving the sensitivity of progressive multiple sequence alignment through sequence weighting, position specific gap penalties and weight matrix choice. Nucleic Acids Res. 22:4673-4680.

30. Troutman, J., and Matejka, J. C. 1970. Fungi associated with cantaloupe in Arizona. (Abstr.) Phytopathology 60:1317.

31. Uecker, F. A., and Pollack, F. G. 1975. Development and cytology of Monosporascus cannonballus. Bot. Gaz. 136:333-340.

32. von Arx, J. A. 1975. On Thielavia angulata and some recently described Thielavia species. Kavaka 3:33-36.

33. Waugh, M. M., Stanghellini, M. E., and Kim, D. 2001. Scanning electron microscopy of germinated ascospores of Monosporascus cannonballus. Mycol. Res. 105:745-748.

34. White, T. J., Bruns, T., Lee, S., and Taylor, J. 1990. Amplification and direct sequencing of fungi ribosomal RNA genes for phylogenetics. Pages 315322 in: PCR Protocols: A Guide to Methods and Applications. Academic Press, San Diego, CA. 